\title{
Pathogen Frequency and Resistance Patterns in Brazilian Hospitals: Summary of Results from Three Years of the SENTRY Antimicrobial Surveillance Program
}

Helio S. Sader, Ana C. Gales, Michael A. Pfaller, Rodrigo E. Mendes, Cássia Zoccoli, Afonso Barth and Ronald N. Jones

\author{
Special Laboratory of Clinical Microbiology, Division \\ of Infectious Diseases, Federal University of São \\ Paulo, SP, Brazil; Anti-Infective Research Center, \\ Department of Pathology, University of Iowa College \\ of Medicine, Iowa City, IA, USA; Santa Luzia Medical \\ Laboratory, Florianópolis, SC, Brazil; Clinical \\ Hospital of Porto Alegre, RS, Brazil.
}

Background: Pathogen frequency and resistance patterns may vary significantly from country to country and also in different hospitals within a country. Thus, regional surveillance programs are essential to guide empirical therapy and infection control measures. Methods: Rank order of occurrence and antimicrobial susceptibility of pathogenic species causing bloodstream infections (BSI), lower respiratory tract infections (LRTI), wound or skin and soft tissue infections (WSSTI), and urinary tract infections (UTI) in hospitalized patients were determined by collecting consecutive isolates over a specified period of time, as part of the SENTRY Antimicrobial Resistance Surveillance Program (SENTRY). All isolates were tested by reference broth microdilution. Results and Conclusions: A total of 3,728 bacterial strains were obtained from January, 1997, to December, 1999, from 12 Brazilian hospitals located in 4 states. The largest number of isolates were obtained from patients with BSI (2,008), followed by LRTI (822 cases), UTI (468 cases), and WSSTI (430 cases). Staphylococcus aureus was the most frequently isolated pathogen in general (22.8\% - 852 isolates), followed by $E$. coli (13.8\% - 516 cases) and Pseudomonas aeruginosa (13.3\% - 496 cases). Staphylococcus aureus was also the most common species isolated from BSI (23.6\%) and WSSTI $(45.8 \%)$, and $P$. aeruginosa was the most frequent species isolated from patients with LRTI (29.4\%). The main bacterial resistance problems found in this study were: imipenem resistance among $P$. aeruginosa $(69.8 \%$ susceptibility) and Acinetobacter spp. $(88.1 \%$ susceptibility); ESBL production among $K$. pneumoniae $(48.4 \%)$ and $E$. coli $(8.9 \%)$; resistance to third generation cephalosporins among Enterobacter spp. (68.1\% susceptible to ceftazidime) and oxacillin resistance among $S$. aureus $(34.0 \%)$ and coagulase negative staphylococci $(80.1 \%)$. Only the carbapenems $(88.1 \%$ to $89.3 \%$ susceptibility) showed reasonable activity against the Acinetobacter spp. isolates evaluated.

Key Words: SENTRY, antimicrobial resistance, nosocomial infection, surveillance program.

Received on 12 April 2001; revised 23 June 2001.

Address for correspondence: Dr. Helio S. Sader, M.D. Laboratório Especial de Microbiologia Clínica, Division of Infectious Diseases, Federal University of São Paulo. Rua Botucatu, 740, Zip code: 04023-900, São Paulo, SP, Brasil. Phone/Fax: 55 (11) 5081-2819/5571-5180/5081-2965.

E-mail: heliosader@uol.com.br

The Brazilian Journal of Infectious Diseases 2001;5(4):200-214 (C) 2001 by The Brazilian Journal of Infectious Diseases and Contexto Publishing. All rights reserved.

$1413-8670$
The euphoria generated by the discovery of antibiotics led to confident predictions that bacterial diseases would soon be conquered and safely forgotten. Scientists felt free to attack other pressing health problems such as viral diseases. However, bacterial pathogens have become increasingly resistant to a variety of antibiotics. This increase in antimicrobial resistance has demanded the development of new and more potent antimicrobial agents and more research in the area of mechanisms of bacterial resistance. A better 
understanding of the dissemination of bacterial resistance to antimicrobial agents is necessary to control the problem.

In general, resistance to increasingly used antimicrobial agents in commonly isolated pathogens is a result of selective pressure caused by the frequent use of these agents [1]. Due to the change in resistance patterns that are occurring in Brazil and worldwide, ongoing surveillance of predominant pathogens and antimicrobial susceptibilities is needed in order to optimize patient care. The SENTRY Antimicrobial Resistance Surveillance Program (SENTRY) started in January, 1997, and was designed to monitor nosocomial and selected community-acquired infections via a worldwide surveillance network of sentinel hospitals distributed equally by geographic location and size [2-4].

This report will focus on the antimicrobial susceptibilities of predominant pathogens causing bloodstream infections, pneumonia, wound infections, and urinary tract infections in Brazil.

\section{Materials and Methods}

\section{Bacterial strains}

SENTRY has enrolled 3 Brazilian sites per year. During 1997 and 1998, the sites were located in Rio de Janeiro, Florianópolis, and São Paulo. In 1999, a site located in the city of Porto Alegre replaced the site located in Rio de Janeiro. Bacterial samples collected between January, 1997, and December, 1999, were evaluated in this study. Only 1 isolate per patient judged to be clinically significant by local criteria was collected. The target numbers of isolates for the evaluated infections to be collected from hospitalized patients at each participating center were: (1) Bloodstream infection (BSI) - 20 consecutive isolates in each calendar month during the 24month period from January 1, 1997, through December 31, 1998; (2) Lower respiratory tract infection (LRTI) - 100 consecutive isolates over a 6-month period each year, from July 1, through December 31; (3) Wound or skin and soft tissue infection (WSSTI) - 50 consecutive isolates over a 3-month period each year, from April 1, 1997, through June 30, 1997; (4) Urinary tract infection (UTI) - 50 consecutive isolates over a 3-month period each year, from February 1, 1997, through May 1, 1997.

\section{Organism identification}

All pathogens were identified at the participating center using routine methods for that laboratory and were confirmed at the coordinating laboratory using automated or conventional methods if needed.

\section{Susceptibility testing}

Antimicrobial susceptibility testing was performed at the coordinating laboratory using broth microdilution methods as described by the National Committee for Clinical Laboratory Standards (NCCLS) [5]. Antimicrobial agents were obtained from respective manufacturers as laboratory grade powder and included macrolides (erythromycin, azithromycin, clarithromycin), the streptogramin quinupristin-dalfopristin, glycopeptides (vancomycin, teicoplanin), fluoroquinolones (gatifloxacin, ciprofloxacin, levofloxacin, trovafloxacin), aminoglycosides (amikacin, gentamicin, tobramycin), carbapenems (imipenem, meropenem), a monobactam (aztreonam), cephalosporins (cefepime, cefuroxime, cefotaxime, ceftriax one, ceftazidime, cefoxitin, cefaclor), penicillins (ampicillin, penicillin, amoxicillin, oxacillin), ß-lactamase inhibitor combinations (amoxicillin-clavulanate, ticarcillinclavulanate, piperacillin-tazobactam), and other drugs including clindamycin, chloramphenicol, tetracycline, rifampin, and trimethoprimsulfamethoxazole.

\section{Quality control}

Quality control was performed utilizing strains from the American type culture collection (ATCC), including S. pneumoniae ATCC 49619, Staphylococcus aureus ATCC 29213, Enterococcus faecalis ATCC 29212, Escherichia coli ATCC 25922, and Pseudomonas aeruginosa ATCC 27853. 


\section{Results}

The species distribution of the pathogens evaluated in this study is summarized in Table 1. A total of 3,728 strains were obtained from the Brazilian centers participating in SENTRY in 1997, 1998, and 1999. Based on the study design, the largest number of isolates were obtained from patients with BSI (2,008 cases), followed by LRTI ( 822 cases), UTI (468 cases), and WSSTI (430).

Table 2 summarizes the susceptibility testing results obtained with the 5 most frequent Gram-negative species listed in the order of occurrence. $P$. aeruginosa was the second most common Gramnegative with almost 500 isolates evaluated (496 strains; $13.3 \%$ ). This pathogen showed extremely high rates of resistance to the majority of the antimicrobial agents tested. The most active compound against this pathogen in Brazil was the carbapenem, meropenem $\left(\mathrm{MIC}_{50}, 1 \mathrm{mg} / \mathrm{mL} ; 74.4 \%\right.$ susceptibility). Ciprofloxacin also showed low MIC results $\left(\mathrm{MIC}_{50}, 0.5 \mathrm{mg} / \mathrm{mL}\right.$ ); however, the percentage of isolates susceptible to this compound was lower (58.7\%). The spectrum rank order of the antimicrobial agents against $P$. aeruginosa in terms of percentage of susceptibility was: meropenem $(74.4 \%)>$ piperacillin/tazobactam
$(70.8 \%)>$ imipenem $(69.8 \%)>$ amikacin $(62.1 \%)>$ cefepime $(59.7 \%)>$ ceftazidime $(59.5 \%)>$ ciprofloxacin $(58.7 \%)$.

The cephalosporins and other b-lactams, except for ampicillin, were active against most of the $E$. coli isolates. Cefepime was the most active of the cephalosporins (95.9\% susceptible) and the carbapenems imipenem and meropenem were the most active b-lactams overall ( $100.0 \%$ susceptible). On the other hand, resistance rates to fluoroquinolones were relatively high with only $89.1 \%$ of strains being susceptible to ciprofloxacin $\left(\mathrm{MIC}_{90},>2 \mathrm{mg} / \mathrm{mL}\right.$ ). Susceptibility to aminoglycosides varied from $97.3 \%$ for amikacin $\left(\mathrm{MIC}_{90}, 8 \mathrm{mg} / \mathrm{mL}\right)$ to $90.5 \%$ for tobramycin $\left(\mathrm{MIC}_{90}, 4 \mathrm{mg} / \mathrm{mL}\right)$.

Antimicrobial resistance rates were much higher among the 318 Klebsiella spp. isolates (Table 2). The carbapenem, imipenem was the most active compound $\left(\mathrm{MIC}_{90}, 0.5 \mathrm{mg} / \mathrm{mL} ; 100 \%\right.$ susceptibility). Although the potency of meropenem $\left(\mathrm{MIC}_{50}, \leq 0.06 \mathrm{mg} / \mathrm{mL}\right)$ was higher than that of imipenem $\left(\mathrm{MIC}_{50}, 0.25 \mathrm{mg} / \mathrm{mL}\right)$, one isolate with an intermediate MIC for meropenem $(8 \mathrm{mg} / \mathrm{mL})$ and susceptible MIC for imipenem $(4 \mathrm{mg} / \mathrm{mL})$ was detected (Table 2). Cefepime showed the highest percentage of susceptible strains $(76.7 \%)$ among b-lactams, other than the carbapenems.

Table 1. Occurrence of the major pathogens isolated in Brazil in 1997, 1998, and 1999

\begin{tabular}{lccccc}
\hline & \multicolumn{5}{c}{ \% Occurrence in } \\
\cline { 2 - 6 } Organisms in rank order & BSI (2008) $^{\mathbf{a}}$ & LRTI (822) $^{\mathbf{b}}$ & SSTI (430) $^{\mathbf{c}}$ & UTI $^{\mathbf{d}} \mathbf{( 4 6 8 )}$ & All sites (3728) \\
\hline S. aureus & 23.6 & 21.0 & 45.8 & 1.9 & $22.8(852)$ \\
E. coli & 11.3 & 4.4 & 7.2 & 47.6 & $13.8(516)$ \\
P. aeruginosa & 7.5 & 29.4 & 10.5 & 12.6 & $13.3(496)$ \\
K. pneumoniae & 8.9 & 9.2 & 4.2 & 9.8 & $8.5(318)$ \\
Enterobacter spp. & 8.3 & 6.8 & 6.7 & 5.8 & $7.5(279)$ \\
CoNS & 12.0 & 0.5 & 3.0 & 0.6 & $7.0(261)$ \\
Acinetobacter spp. & 6.8 & 10.8 & 2.8 & 3.0 & $6.7(252)$ \\
Enterococcus spp. & 2.7 & 4.0 & 8.4 & 5.1 & $4.0(147)$ \\
Serratia spp. & 2.5 & 3.3 & 2.8 & 2.7 & $2.7(102)$ \\
Proteus spp. & 0.7 & - & 3.5 & 5.1 & $1.4(54)$ \\
\hline
\end{tabular}

${ }^{a}$ BSI: blood stream infection; ${ }^{\text {b }}$ LRTI: lower respiratory tract infection; ${ }^{c}$ WSSTI: wound or skin and soft tissue infection; ${ }^{\text {d }}$ UTI: urinary tract infection; ${ }^{\text {e }}$ CoNS: Coagulase-negative staphylococci. 
Table 2. Antimicrobial susceptibility pattern of the most frequent Gram-negative bacilli collected from hospitalized patients in Brazil - SENTRY Program 1997, 1998, and 1999

\begin{tabular}{|c|c|c|c|c|c|c|c|c|c|c|}
\hline \multirow[t]{3}{*}{ Antimicrobial class/agent } & \multicolumn{10}{|c|}{ Pathogen (prevalence rank/ no. tested) } \\
\hline & \multicolumn{2}{|c|}{ E. $\operatorname{coli}(2 / 516)$} & \multicolumn{2}{|c|}{ P. aeruginosa (3/496) } & \multicolumn{2}{|c|}{ K. pneumoniae. (4/318) } & \multicolumn{2}{|c|}{ Enterobacter spp.(5/279) } & \multicolumn{2}{|c|}{ Acinetobacter spp. $(7 / 252$} \\
\hline & $\mathrm{MIC}_{50} /_{90}$ & $\%$ Susc. $^{a}$ & $\mathrm{MIC}_{50} \mathrm{I}_{9}$ & $\% \operatorname{Susc}^{a}$ & $\mathrm{MIC}_{50} / 90$ & $\% \operatorname{Susc}^{a}$ & $\mathrm{MIC}_{50} \mathbf{l}_{90}$ & $\%$ Susc $^{a}$ & $\mathrm{MIC}_{50} J_{90}$ & $\%$ Susc $^{\mathrm{a}}$ \\
\hline \multicolumn{11}{|l|}{ Cephalosporins } \\
\hline Cefazolin & $\leq 2 />16$ & 84.1 & $>16 />16$ & 0.0 & $>16 />16$ & 45.0 & $>16 />16$ & 2.9 & $>16 />16$ & 0.4 \\
\hline Cefuroxime & $4 / 16$ & 87.0 & $>16 />16$ & 0.0 & $8 />16$ & 50.6 & $>16 />16$ & 37.3 & $>16 />16$ & 3.6 \\
\hline Cefoxitin & $4 / 8$ & 93.6 & $>32 />32$ & 0.2 & $4 / 16$ & 84.3 & $>32 />32$ & 5.4 & $>32 />32$ & 1.2 \\
\hline Ceftriaxone & $\leq 0.25 / \leq 0.25$ & $92.6(7.8)^{\mathrm{b}}$ & $>32 />32$ & 4.8 & $\leq 0.25 />32$ & $58.8(48.4)^{\mathrm{b}}$ & $0.5 />32$ & 69.5 & $>32 />32$ & 12.7 \\
\hline Ceftazidime & $0.25 / 1$ & $94.6(8.9)^{\mathrm{b}}$ & $8 />16$ & 59.5 & $1 />16$ & $68.2(48.4)^{\mathrm{b}}$ & $0.5 />16$ & 68.1 & $>16 />16$ & 26.6 \\
\hline Cefepime & $\leq 0.12 / 0.25$ & 95.9 & $8 />16$ & 59.7 & $0.25 />16$ & 76.7 & $\leq 0.12 / 8$ & 91.4 & $>16 />16$ & 34.1 \\
\hline \multicolumn{11}{|l|}{ Other b-lactams } \\
\hline Ampicillin & $>16 />16$ & 42.2 & $>16 />16$ & 0.2 & $>16 />16$ & 2.5 & $>16 />16$ & 5.7 & $>16 />16$ & 4.4 \\
\hline Aztreonam & $\leq 0.12 / 0.25$ & $93.2(8.5)^{b}$ & $16 />16$ & 42.7 & $0.25 />16$ & $59.7(45.6)^{\mathrm{b}}$ & $0.25 />16$ & 68.5 & $>16 />16$ & 6.7 \\
\hline Ticarcillin/Clavulanate & $8 / 128$ & 69.4 & $64 />128$ & 52.0 & $32 />128$ & 48.7 & $8 />128$ & 54.5 & $128 />128$ & 28.6 \\
\hline Piperacillin/Tazobactam & $2 / 16$ & 92.4 & $16 />64$ & 70.8 & $4 />64$ & 67.0 & $4 />64$ & 67.4 & $>64 />64$ & 30.2 \\
\hline Imipenem & $0.12 / 0.5$ & 100.0 & $2 />8$ & 69.8 & $0.25 / 0.5$ & 100.0 & $0.5 / 2$ & 99.6 & $1 />8$ & 88.1 \\
\hline Meropenem & $\leq 0.06 / \leq 0.06$ & 100.0 & $1 />8$ & 74.4 & $\leq 0.06 / 0.12$ & 99.7 & $\leq 0.06 / 0.25$ & 100.0 & $1 />8$ & 89.3 \\
\hline \multicolumn{11}{|l|}{ Aminoglycosides } \\
\hline Amikacin & $4 / 8$ & 97.3 & $8 />32$ & 62.1 & $2 />32$ & 79.2 & $2 />32$ & 82.4 & $>32 />32$ & 31.7 \\
\hline Gentamicin & $1 / 4$ & 92.6 & $4 />16$ & 55.6 & $1 />16$ & 64.8 & $1 />16$ & 77.4 & $16 />16$ & 49.6 \\
\hline Tobramycin & $1 / 4$ & 90.5 & $1 />16$ & 55.8 & $1 />16$ & 55.7 & $1 />16$ & 69.2 & $4 />16$ & 56.3 \\
\hline \multicolumn{11}{|l|}{ Fluoroquinolones } \\
\hline Ciprofloxacin & $0.03 />2$ & 89.1 & $0.5 />2$ & 58.7 & $0.06 / 1$ & 94.3 & $0.06 />2$ & 83.2 & $>2 />2$ & 35.3 \\
\hline Gatifloxacin & $\leq 0.03 / 2$ & 90.1 & $2 />4$ & 55.0 & $0.06 / 1$ & 95.6 & $0.06 / 4$ & 86.4 & $4 />4$ & 40.5 \\
\hline Levofloxacin & $0.5 / 4$ & 89.5 & $1 />4$ & 57.1 & $0.5 / 1$ & 94.7 & $0.5 />4$ & 84.9 & $4 />4$ & 36.9 \\
\hline Trovafloxacin & $\leq 0.03 / 4$ & 89.1 & $4 />4$ & 52.6 & $0.06 / 1$ & 93.4 & $0.06 />4$ & 81.7 & $4 />4$ & 43.7 \\
\hline Grepafloxacin & $\leq 0.25 />2$ & 88.9 & $1 />2$ & 54.0 & $\leq 0.25 / 0.5$ & 93.5 & $\leq 0.25 />2$ & 78.9 & $>2 />2$ & 39.4 \\
\hline \multicolumn{11}{|l|}{ Others } \\
\hline Tetracycline & $\leq 4 />8$ & 59.5 & $>8 />8$ & 1.0 & $\leq 4 />8$ & 73.9 & $\leq 4 />8$ & 66.7 & $\leq 4 />8$ & 64.7 \\
\hline $\begin{array}{l}\text { Trimethoprim/ } \\
\text { Sulfathoxazole }\end{array}$ & $\leq 0.5 />2$ & 53.7 & $2 />2$ & 3.2 & $\leq 0.5 />1$ & 65.6 & $\leq 0.5 />2$ & 72.0 & $2 />2$ & 32.1 \\
\hline
\end{tabular}

a Percentage of susceptible strains according to NCCLS criteria [NCCLS 2000; ${ }^{\text {b }}$ Percentage of strains with MIC $\geq 2 \mathrm{mg} / \mathrm{mL}$ indicating possible ESBL production. 
Table 3. Percentages of E. coli and K. pneumoniae considered ESBL producers based on the NCCLS criteria.

\begin{tabular}{lrccc}
\hline Site of infection & \multicolumn{3}{c}{$\%$ ESBL Producing strains (n/total) } \\
\cline { 2 - 5 } & \multicolumn{2}{c}{ E. coli } & K. pneumoniae \\
\hline Bacteremia & $8.4 \%$ & $(19 / 226)$ & $52.2 \%$ & $(93 / 178)$ \\
Pneumonia & $30.6 \%$ & $(11 / 36)$ & $48.7 \%$ & $(37 / 76)$ \\
Urinary tract & $5.8 \%$ & $(13 / 223)$ & $45.6 \%$ & $(21 / 46)$ \\
Wound, skin, and soft tissue & $12.9 \%$ & $(4 / 31)$ & $50.0 \%$ & $(9 / 18)$ \\
Total & $\mathbf{9 . 1 \%}$ & $(\mathbf{4 7 / 5 1 6})$ & $\mathbf{5 0 . 3 \%}$ & $(\mathbf{1 6 0 / 3 1 8})$
\end{tabular}

a Strains with increased MICs $(\geq 2 \mathrm{mg} / \mathrm{mL}$ ) for ceftazidime, aztreonam, ceftriaxone or cefotaxime indicating possible ESBL production.

Table 4. Antimicrobial susceptibility E.coli and K. pneumoniae strains classified as ESBL-producers based on the NCCLS criteria

\begin{tabular}{|c|c|c|c|c|}
\hline \multirow[t]{3}{*}{ Antimicrobial class/agent } & \multicolumn{4}{|c|}{ Pathogens (\%) } \\
\hline & \multicolumn{2}{|c|}{ E. $\operatorname{coli}^{\text {a }}(47)$} & \multicolumn{2}{|c|}{ K. pneumoniae ${ }^{a}(160)$} \\
\hline & $\mathrm{MIC}_{50} / 90$ & $\%$ Susc. & $\mathrm{MIC}_{50} / 90$ & $\%$ Susc. \\
\hline \multicolumn{5}{|l|}{ b-lactams } \\
\hline Cefoxitin & $4 / 32$ & 76.6 & $4 / 32$ & 78.1 \\
\hline Ticarcillin/Clavulanate & $128 />128$ & 8.5 & $128 />128$ & 8.1 \\
\hline Piperacillin/ Tazobactam & $16 />64$ & 51.1 & $64 />64$ & 39.4 \\
\hline Imipenem & $0.25 / 0.5$ & 100.0 & $0.25 / 0.5$ & 100.0 \\
\hline Meropenem & $\leq 0.06 / 0.12$ & 100.0 & $\leq 0.06 / 0.12$ & 99.4 \\
\hline \multicolumn{5}{|l|}{ Aminoglycosides } \\
\hline Amikacin & $16 / 32$ & 74.5 & $16 />32$ & 63.1 \\
\hline Gentamicin & $16 />16$ & 36.2 & $16 />16$ & 36.9 \\
\hline Tobramicin & $>16 />16$ & 19.1 & $>16 />16$ & 19.4 \\
\hline \multicolumn{5}{|l|}{ Fluoroquinolones } \\
\hline Ciprofloxacin & $0.25 />2$ & 70.2 & $0.25 / 1$ & 90.6 \\
\hline Levofloxacin & $0.5 />4$ & 70.2 & $0.5 / 2$ & 91.3 \\
\hline Gatifloxacin & $\leq 0.03 />4$ & 74.5 & $0.06 / 2$ & 92.5 \\
\hline \multicolumn{5}{|l|}{ Others } \\
\hline Tetracycline & $>8 />8$ & 40.4 & $\leq 4 />8$ & 66.3 \\
\hline Trimethoprim/Sulfathoxazole & $>1 />1$ & 27.7 & $>1 />1$ & 46.3 \\
\hline
\end{tabular}


Table 5. Antimicrobial susceptibility pattern of the most frequent Gram-positive cocci from hospitalized patients in Brazil - SENTRY Program 1997, 1998, and 1999

\begin{tabular}{|c|c|c|c|c|c|c|}
\hline \multirow[t]{2}{*}{ Antimicrobial class/agent } & \multicolumn{6}{|c|}{ Pathogen (prevalence rank/ no. tested) } \\
\hline & \multicolumn{2}{|c|}{ S. aureus (1/852) } & \multicolumn{2}{|c|}{$\operatorname{CoNS}^{\mathrm{a}}(6 / 261)$} & \multirow[t]{2}{*}{ Enterococcus } & \multirow{2}{*}{ spp. $(8 / 147)$} \\
\hline \multicolumn{5}{|l|}{ Cephalosporins } & & \\
\hline Cefazolin & $\leq 2 />16$ & $66.0^{\mathrm{b}}$ & $4 />16$ & $19.9^{\mathrm{b}}$ & $>16 />16$ & - \\
\hline Ceftriaxone & $4 />32$ & $66.0^{\mathrm{b}}$ & $16 />32$ & $19.9^{\mathrm{b}}$ & $>32 />32$ & - \\
\hline Cefepime & $4 />16$ & $66.0^{\mathrm{b}}$ & $8 />16$ & $19.9^{b}$ & $>16 />16$ & - \\
\hline Ceftazidime & $8 />16$ & 51.8 & $>16 />16$ & $19.9^{b}$ & $>16 />16$ & - \\
\hline \multicolumn{7}{|l|}{ Other b-lactams } \\
\hline Oxacilliin & $0.5 />8$ & 66.0 & $>8 />8$ & 19.9 & $>8 />8$ & - \\
\hline Ampicillin & $16 />16$ & 9.9 & $16 />16$ & 12.6 & $1 / 4$ & 98.0 \\
\hline Penicillin & $16 />32$ & 9.2 & $16 />32$ & 8.1 & $2 / 16$ & 89.1 \\
\hline Amoxicillin/Clavulanate & $2 />16$ & 68.1 & $4 />16$ & $19.9^{\mathrm{b}}$ & $1 / 2$ & $89.1^{\mathrm{c}}$ \\
\hline Piperacillin/Tazobactam & $2 />64$ & $66.0^{\mathrm{b}}$ & $4 />64$ & $19.9^{\mathrm{b}}$ & $4 />64$ & $89.1^{\mathrm{c}}$ \\
\hline Imipenem & $\leq 0.06 />8$ & $66.0^{\mathrm{b}}$ & $1 />8$ & $19.9^{\mathrm{b}}$ & $2 / 8$ & - \\
\hline \multicolumn{7}{|l|}{ MLS } \\
\hline Clindamycin & $0.25 />8$ & 66.5 & $>8 />8$ & 46.4 & $>8 />8$ & - \\
\hline Erythromycin & $1 />8$ & 45.3 & $>8 />8$ & 39.1 & $>8 />8$ & 6.1 \\
\hline Doxycycline & $0.5 />8$ & 81.0 & $1 / 8$ & 85.8 & $4 / 8$ & 55.1 \\
\hline \multicolumn{7}{|l|}{ Fluoroquinilones } \\
\hline Ciprofloxacin & $0.5 />2$ & 65.6 & $2 />2$ & 49.8 & $2 />2$ & 49.0 \\
\hline Gatifloxacin & $0.12 / 4$ & 89.3 & $0.5 / 2$ & 92.7 & $0.5 />4$ & 73.5 \\
\hline Trovafloxacin & $\leq 0.03 / 1$ & 90.3 & $0.25 / 4$ & 75.9 & $0.25 />4$ & 72.1 \\
\hline \multicolumn{7}{|l|}{ Others } \\
\hline Gentamicin & $1 />16$ & 64.8 & $16 />16$ & 41.4 & $16 />16$ & 27.2 \\
\hline $\operatorname{Gentamicin}(H L)^{d}$ & $\leq 500 / \leq 500$ & - & $\leq 500 / \leq 500$ & - & $\leq 500 />1000$ & 72.8 \\
\hline Streptomycin $(\mathrm{HL})^{\mathrm{d}}$ & $\leq 1000 / 2000$ & - & $\leq 1000 / 2000$ & - & $\leq 1000 />2000$ & 70.1 \\
\hline Rifampin & $0.25 / 2$ & 71.1 & $0.25 />2$ & 67.8 & $>2 />2$ & 20.4 \\
\hline Chloramphenicol & $8 />16$ & 58.8 & $8 />16$ & 50.6 & $8 />16$ & 60.5 \\
\hline Tetracycline & $\leq 4 />8$ & 62.3 & $\leq 4 />8$ & 75.1 & $>8 />8$ & 35.4 \\
\hline \multicolumn{7}{|l|}{ Trimethoprim/ } \\
\hline Sulfametoxale & $\leq 0.5 />2$ & 68.0 & $2 />2$ & 40.2 & $\leq 0.5 / 2$ & 76.9 \\
\hline Quinupristin/dalfopristin & $0.25 / 0.5$ & 99.8 & $0.25 / 1$ & 98.9 & $8 />8$ & 3.4 \\
\hline Teicoplanin & $1 / 2$ & 99.8 & $2 / 16$ & 88.9 & $0.25 / 0.5$ & 99.3 \\
\hline Vancomycin & $1 / 1$ & 100.0 & $1 / 2$ & 100.0 & $1 / 2$ & 99. \\
\hline
\end{tabular}

${ }^{a}$ CoNS: Coagulase-negative staphylococci.

${ }^{\mathrm{b}}$ Susceptibility predicted by the oxacillin result.

${ }^{c}$ Susceptibility predicted by the penicillin result.

${ }^{\mathrm{d}}$ High level aminoglycoside resistance screen. 
Table 6. Antimicrobial susceptibility pattern of oxacillin-resistant Staphylococcus aureus strains from hospitalized patients in Brazil

\begin{tabular}{lccc}
\hline Antimicrobial agent & \multicolumn{3}{c}{ Oxacillin-resistant Staphylococcus aureus (290) } \\
\cline { 2 - 4 } & $\mathbf{M I C}_{\mathbf{5 0}}(\mathbf{m g} / \mathbf{m L})$ & $\mathbf{M I C}_{\mathbf{9 0}}(\mathbf{m g} / \mathbf{m L})$ & \% Susceptible \\
\hline Clindamycin & $>8$ & $>8$ & 7.2 \\
Erythromycin & $>$ & $>8$ & 2.4 \\
Doxycycline & 8 & 8 & 47.9 \\
Ciprofloxacin & $>2$ & $>2$ & 7.2 \\
Gatifloxacin & 2 & 4 & 69.3 \\
Gentamicin & $>16$ & $>16$ & 4.5 \\
Rifampin & 2 & $>2$ & 20.3 \\
Chloramphenicol & $>16$ & $>8$ & 21.7 \\
Tetracycline & $>8$ & $>1$ & 10.3 \\
Trimethoprim/sulfamethoxale & $>1$ & 1 & 8.3 \\
Quinupristin/dalfopristin & 0.5 & 2 & 99.3 \\
Teicoplanin & 2 & 1 & 99.3 \\
Vancomycin & 1 & & 100.0 \\
\hline
\end{tabular}

The percentage of E. coli and K. pneumoniae producing ESBLs remains very high in Brazil. Utilizing the screening concentrations recommended by the NCCLS [5] to predict isolates of $K$. pneumoniae and $E$. coli suspected of harboring ESBLs (MICs $\geq 2 \mathrm{mg} / \mathrm{mL}$ to ceftazidime or aztreonam or ceftriaxone), $9.1 \%$ of $E$. coli and $50.3 \%$ of Klebsiella met these criteria (Table 3 ).

Table 4 summarizes the antimicrobial susceptibility results of the ESBL-producing strains. Only the carbapenems showed excellent activitiy against these pathogens. ESBL-producing strains also showed high rates of resistance to cefoxitin, a cefamycin stable to hydrolysis by ESBLs; and to piperacillin/tazobactam, a b-lactam-b-lactamases inhibitor combination. Cefoxitin was active against only $78.1 \%$ of the $K$. pneumoniae and $76.6 \%$ of the $E$. coli isolates at $£ 8$ $\mathrm{mg} / \mathrm{mL}$, suggesting that other mechanisms of resistance, such as the production of $A m p C$ enzymes (strains with cefoxitin MICs, $\geq 32 \mathrm{mg} / \mathrm{mL}$ ) and/or alteration in the outer membrane might be associated to the ESBL production. Ticarcillin/clavulanic acid was active against only $8.1 \%$ of the $K$. pneumoniae $\left(\mathrm{MIC}_{90},>128 \mathrm{mg} /\right.$ $\mathrm{mL})$ and $8.5 \%$ of the $E$. coli isolates $\left(\mathrm{MIC}_{90},>128\right.$ $\mathrm{mg} / \mathrm{mL}$ ). Piperacillin/tazobactam showed in vitro activity higher than that of ticarcillin/clavulanic acid, but only $39.4 \%$ of $K$. pneumoniae $\left(\mathrm{MIC}_{90},>64 \mathrm{mg} / \mathrm{mL}\right.$ ) and $51.1 \%$ of E. coli $\left(\mathrm{MIC}_{90},>64 \mathrm{mg} / \mathrm{mL}\right)$ were susceptible to this compound. The fluoroquinolones were very active against ESBL-producing $K$. pneumoniae ( $>90 \%$ susceptibility), but not against $E$. coli isolates (70.2\% to $74.5 \%$ susceptibility).

Enterobacter spp. (279 isolates analyzed) showed high rates of resistance to broad spectrum penicillins with or without b-lactamase inhibitors $(67.4 \%$ susceptibility to piperacillin/tazobactam) and thirdgeneration cephalosporins ( $68.1 \%$ susceptibility to ceftazidime). However, cefepime ( $91.4 \%$ susceptibility) remains very active against this pathogen in Brazil. Aminoglycoside resistance was also elevated among Enterobacter spp. (69.2 to $82.4 \%$ susceptibility), and the most active fluoroquinolone against this pathogen was gatifloxacin ( $86.4 \%$ susceptibility).

Acinetobacter spp. was the fifth most frequent Gram-negative bacilli genus isolated in general (6.7\% of the isolates), and the third most common pathogen isolated from hospitalized patients with pneumonia (10.8\% of the isolates, Table 1). In our study, the carbapenems were active against nearly $90 \%$ of Acinetobacter spp. isolates $\left(\mathrm{MIC}_{50}, 1 \mathrm{mg} / \mathrm{mL}\right.$ and 
Table 7. Antimicrobial activity and spectrum of drugs tested against the 5 most prevalent Gram-negative pathogens causing bloodstream infections in Brazil

\begin{tabular}{|c|c|c|c|c|c|c|c|c|c|c|}
\hline \multirow[t]{3}{*}{ Antimicrobial class/agent } & \multicolumn{10}{|c|}{ Pathogen (prevalence rank/ $\mathbf{n}^{\circ}$ tested) } \\
\hline & \multicolumn{2}{|c|}{ E. $\operatorname{coli}(3 / 226)$} & \multicolumn{2}{|c|}{ K. pneumoniae. $(4 / 178)$} & \multicolumn{2}{|c|}{ Enterobacter spp.(5/167) } & \multicolumn{2}{|c|}{ P. aeruginosa $(6 / 150)$} & \multicolumn{2}{|c|}{ Acinetobacter spp.(7/137) } \\
\hline & $\mathrm{MIC}_{50} \mathrm{I}_{90}$ & \% Susc. ${ }^{a}$ & $\mathrm{MIC}_{50} / 90$ & $\%$ Susc $^{a}$ & $\mathrm{MIC}_{50} J_{90}$ & $\%$ Susc $^{a}$ & $\mathrm{MIC}_{50} /_{90}$ & $\%$ Susc $^{a}$ & $\mathrm{MIC}_{50} / 90$ & $\%$ Susc $^{a}$ \\
\hline \multicolumn{11}{|l|}{ Cephalosporins } \\
\hline Cefazolin & $\leq 2 />16$ & 82.7 & $>16 />16$ & 42.1 & $>16 />16$ & 2.4 & $>16 />16$ & 0.0 & $>16 />16$ & 0.0 \\
\hline Cefuroxime & $16 />16$ & 40.7 & $16 />16$ & 49.4 & $16 />16$ & 40.7 & $>16 />16$ & 0.0 & $>16 />16$ & 6.6 \\
\hline Cefoxitin & $4 / 8$ & 93.4 & $4 / 16$ & 86.5 & $>32 />32$ & 4.8 & $>32 />32$ & 0.7 & $>32 />32$ & 1.5 \\
\hline Ceftriaxone & $\leq 0.25 / 0.5$ & $92.0(8.0)^{b}$ & $2 />32$ & $56.2(51.1)^{b}$ & $\leq 0.25 />32$ & 74.3 & $>32 />32$ & 6.7 & $>32 />32$ & 16.1 \\
\hline Ceftazidime & $0.25 / 1$ & $93.8(8.4)^{\mathrm{b}}$ & $1 />16$ & $69.1(48.4)^{b}$ & $0.25 />16$ & 73.7 & $4 />16$ & 66.7 & $>16 />16$ & 38.0 \\
\hline Cefepime & $\leq 0.12 / 0.25$ & 96.0 & $0.25 />16$ & 74.7 & $\leq 0.12 / 8$ & 91.0 & $4 />16$ & 66.7 & $16 />16$ & 44.5 \\
\hline \multicolumn{11}{|l|}{ Other b-lactams } \\
\hline Ampicillin & $>16 />16$ & 39.4 & $>16 />16$ & 4.5 & $>16 />16$ & 6.0 & $>16 />16$ & 0.0 & $>16 />16$ & 6.6 \\
\hline Aztreonam & $\leq 0.12 / 0.25$ & $92.9(8.4)^{\mathrm{b}}$ & $0.5 />16$ & $59.6(47.2)^{\mathrm{b}}$ & $\leq 0.12 />16$ & 74.9 & $8 />16$ & 52.7 & $>16 />16$ & 10.9 \\
\hline Ticarcillin/Clavulanate & $8 / 128$ & 68.1 & $32 />128$ & 47.8 & $4 />128$ & 59.9 & $64 />128$ & 64.0 & $64 />128$ & 39.4 \\
\hline \multicolumn{11}{|l|}{ Piperacillin/ } \\
\hline Tazobactam & $2 / 16$ & 91.2 & $4 />64$ & 68.0 & $2 />64$ & 70.7 & $8 />64$ & 74.0 & $64 />64$ & 40.1 \\
\hline Imipenem & $0.12 / 0.25$ & 100.0 & $0.25 / 0.5$ & 100.0 & $0.5 / 2$ & 99.4 & $2 />8$ & 82.0 & $0.5 / 4$ & 90.5 \\
\hline Meropenem & $\leq 0.06 / \leq 0.06$ & 100.0 & $\leq 0.06 / 0.12$ & 100.0 & $\leq 0.06 / 0.25$ & 100.0 & $0.5 />8$ & 82.7 & $1 / 4$ & 92.0 \\
\hline \multicolumn{11}{|l|}{ Aminoglycosides } \\
\hline Amikacin & $4 / 8$ & 96.5 & $2 />32$ & 75.8 & $2 />32$ & 81.4 & $4 />32$ & 70.0 & $>32 />32$ & 42.3 \\
\hline Gentamicin & $1 / 2$ & 91.6 & $1 />16$ & 60.7 & $1 />16$ & 75.4 & $2 />16$ & 62.0 & $4 />16$ & 51.8 \\
\hline Tobramycin & $1 / 4$ & 90.7 & $2 />16$ & 52.8 & $1 />16$ & 70.7 & $1 />16$ & 60.7 & $2 />16$ & 67.9 \\
\hline \multicolumn{11}{|l|}{ Fluoroquinolones } \\
\hline Ciprofloxacin & $0.03 / 0.5$ & 91.6 & $0.06 / 0.5$ & 95.5 & $0.06 />2$ & 86.2 & $0.25 />2$ & 67.3 & $>2 />2$ & 44.5 \\
\hline Gatifloxacin & $\leq 0.03 / 0.5$ & 92.5 & $0.06 / 0.5$ & 96.6 & $0.06 / 4$ & 88.6 & $1 />4$ & 66.7 & $4 />4$ & 48.2 \\
\hline Levofloxacin & $0.5 / 0.5$ & 92.0 & $0.5 / 1$ & 94.9 & $0.5 / 4$ & 87.4 & $0.5 />4$ & 68.0 & $4 />4$ & 47.4 \\
\hline Trovafloxacin & $\leq 0.03 / 0.5$ & 91.6 & $0.06 / 0.5$ & 94.9 & $0.06 / 4$ & 84.4 & $0.5 />4$ & 64.0 & $1 />4$ & 50.4 \\
\hline Grepafloxacin & $\leq 0.25 / \leq 0.25$ & 95.8 & $\leq 0.25 / 0.5$ & 92.6 & $\leq 0.25 />2$ & 80.6 & $0.5 />2$ & 65.7 & $\leq 0.25 />2$ & 51.7 \\
\hline \multicolumn{11}{|l|}{ Others } \\
\hline Tetracycline & $\leq 4 />8$ & 60.2 & $\leq 4 />8$ & 78.7 & $\leq 4 />8$ & 70.7 & $>8 />8$ & 1.3 & $\leq 4 />8$ & 70.1 \\
\hline Trimethoprim/ & & & & & & & & & & \\
\hline Sulfathoxazole & $2 />2$ & 46.9 & $\leq 0.5 />2$ & 61.0 & $\leq 0.5 />2$ & 73.7 & $2 />2$ & 4.0 & $2 />2$ & 38.7 \\
\hline
\end{tabular}

Percentage of susceptible strains according to NCCLS criteria [NCCLS 2000].

${ }^{\mathrm{b}}$ Percentage of strains with MIC $\geq 2 \mathrm{mg} / \mathrm{mL}$ indicating possible ESBL production. 
Table 8. Antimicrobial activity and spectrum of drugs tested against the most prevalent Gram-positive pathogens causing bloodstream infections in Brazil - SENTRY program 1997 to 1999

\begin{tabular}{|c|c|c|c|c|c|c|}
\hline \multirow[b]{2}{*}{ Antimicrobial class/agent } & \multicolumn{6}{|c|}{ Pathogen (prevalence rank/ no. tested) } \\
\hline & \multicolumn{2}{|c|}{ S. aureus $(1 / 473)$} & \multicolumn{2}{|c|}{$\operatorname{CoNS}^{a}(2 / 241)$} & \multicolumn{2}{|c|}{ Enterococcus spp. $(8 / 54)$} \\
\hline \multicolumn{7}{|l|}{ Cephalosporins } \\
\hline Cefazolin & $\leq 2 />16$ & $70.6^{\mathrm{b}}$ & $4 />16$ & $19.9^{\mathrm{b}}$ & $>16 />16$ & - \\
\hline Ceftriaxone & $4 />32$ & $70.6^{\mathrm{b}}$ & $16 />32$ & $19.9^{\mathrm{b}}$ & $>32 />32$ & - \\
\hline Cefepime & $4 />16$ & $70.6^{\mathrm{b}}$ & $8 />16$ & $19.9^{\mathrm{b}}$ & $>16 />16$ & - \\
\hline Ceftazidime & $8 />16$ & 55.1 & $>16 />16$ & $19.9^{\mathrm{b}}$ & $>16 />16$ & - \\
\hline \multicolumn{7}{|l|}{ Other b-lactams } \\
\hline Oxacillin & $0.5 />8$ & 70.6 & $>8 />8$ & 19.9 & $>8 />8$ & - \\
\hline Ampicillin & $16 />16$ & 12.3 & $16 />16$ & 13.3 & $1 / 4$ & 94.4 \\
\hline Penicillin & $16 />32$ & 11.6 & $16 />32$ & 8.3 & $4 / 16$ & 85.2 \\
\hline Amoxicillin/Clavulanate & $2 />16$ & $70.6^{\mathrm{b}}$ & $4 />16$ & $19.9^{b}$ & $1 / 4$ & $85.2^{\mathrm{c}}$ \\
\hline Piperacillin/Tazobactam & $2 />64$ & $70.6^{\mathrm{b}}$ & $4 />64$ & 19.9 & $4 />64$ & $85.2^{\mathrm{c}}$ \\
\hline Imipenem & $\leq 0.06 />8$ & $70.6^{\mathrm{b}}$ & $1 />8$ & 19.9 & $2 / 8$ & - \\
\hline \multicolumn{7}{|l|}{ MLS } \\
\hline Clindamycin & $0.25 />8$ & 70.8 & $>8 />8$ & 45.6 & $>8 />8$ & - \\
\hline Erythromycin & $1 />8$ & 49.5 & $>8 />8$ & 39.4 & $>8 />8$ & 1.9 \\
\hline Doxycycline & $0.5 />4$ & 84.3 & $1 / 8$ & 86.7 & $4 / 8$ & 63.0 \\
\hline \multicolumn{7}{|l|}{ Fluoroquinolones } \\
\hline Ciprofloxacin & $0.5 />2$ & 70.6 & $1 />2$ & 50.2 & $2 />2$ & 38.9 \\
\hline Gatifloxacin & $0.12 / 4$ & 89.0 & $0.25 / 2$ & 92.9 & $0.5 />4$ & 66.7 \\
\hline Trovafloxacin & $\leq 0.03 / 1$ & 90.7 & $0.25 / 4$ & 75.9 & $0.25 />4$ & 64.8 \\
\hline \multicolumn{7}{|l|}{ Others } \\
\hline Gentamicin & $1 />16$ & 68.5 & $16 />16$ & 41.1 & $16 />16$ & - \\
\hline Gentamicin $(H L)^{d}$ & $\leq 500 / \leq 500$ & - & $\leq 500 / \leq 500$ & - & $\leq 500 />1000$ & 63.0 \\
\hline Streptomycin $(\mathrm{HL})^{\mathrm{d}}$ & $\leq 1000 / 2000$ & - & $\leq 1000 / 2000$ & - & $\leq 1000 />2000$ & 68.5 \\
\hline Rifampin & $0.5 / 2$ & 77.8 & $0.25 />2$ & 68.0 & $>2 />2$ & 20.4 \\
\hline Chloramphenicol & $8 />16$ & 63.6 & $16 />16$ & 49.8 & $8 />16$ & 53.7 \\
\hline Tetracycline & $\leq 4 />8$ & 66.6 & $\leq 4 />8$ & 76.3 & $>8 />8$ & 37.0 \\
\hline \multicolumn{7}{|l|}{ Trimethoprim/ } \\
\hline Sulfamethoxazole & $\leq 0.5 / 2$ & 72.7 & $2 />2$ & 39.0 & $\leq 0.5 / 2$ & 68.5 \\
\hline Quinn/Dalf. & $0.25 / 0.5$ & 99.6 & $0.25 / 1$ & 98.8 & $4 />8$ & 5.6 \\
\hline Teicoplanin & $1 / 2$ & 99.6 & $2 / 16$ & 88.4 & $\leq 0.12 / 0.5$ & 98.1 \\
\hline Vancomycin & $1 / 1$ & 100.0 & $2 / 2$ & 100.0 & $1 / 2$ & 98.1 \\
\hline
\end{tabular}

a CoNS: coagulase-negative staphylococci.

${ }^{\mathrm{b}}$ Susceptibility predicted by the oxacillin result.

c Susceptibility predicted by the penicillin result.

${ }^{d}$ High level aminoglycoside resistence screen. 
Table 9. Comparison of the antimicrobial susceptibility of Pseudomonas aeruginosa isolates collected from various infection sites

\begin{tabular}{lccccc}
\hline Antimicrobial agents & \multicolumn{5}{c}{ \% Susceptible } \\
\cline { 2 - 6 } & BSI $^{\mathbf{a}}$ (150) & LRTI $^{\mathbf{b}}$ ( 242) & WSSTI $^{\mathbf{c}} \mathbf{( 4 5 )}$ & UTI $^{\mathbf{d}}$ (59) & All sites (496) \\
\hline Cefepime & 66.7 & 59.9 & 55.6 & 44.1 & 59.7 \\
Ceftazidime & 66.7 & 59.5 & 55.6 & 44.1 & 59.5 \\
Piperacillin/tazobactam & 74.0 & 70.7 & 71.1 & 62.7 & 70.8 \\
Imipenem & 82.0 & 66.9 & 64.4 & 54.2 & 69.8 \\
Meropenem & 82.7 & 71.1 & 75.6 & 66.1 & 74.4 \\
Gentamicin & 62.0 & 55.0 & 62.2 & 25.4 & 55.6 \\
Amikacin & 70.0 & 61.6 & 68.9 & 39.0 & 55.8 \\
Ciprofloxacin & 67.3 & 59.9 & 57.8 & 32.2 & 58.7 \\
Gatifloxacin & 66.7 & 54.1 & 51.1 & 32.2 & 55.0 \\
\hline
\end{tabular}

${ }^{a}$ BSI: bloodstream infection.

b LRTI: lower respiratory tract infection.

${ }^{c}$ WSSTI: wound or skin and soft tissue infection.

${ }^{\mathrm{d}}$ UTI: urinary tract infection.

Table 10. Comparison of the antimicrobial susceptibility of Enterobacter spp. isolates collected from various infection sites

\begin{tabular}{lccccc}
\hline Antimicrobial agents & \multicolumn{4}{c}{ \% Susceptible } \\
\cline { 2 - 6 } & BSI $^{\mathbf{a}}$ (167) & LRTI $^{\mathbf{b}}$ (56) & WSSTI $^{\mathbf{c}}$ (29) & UTI $^{\mathbf{d}}$ (27) & All sites (279) \\
\hline Cefepime & 91.0 & 89.3 & 100.0 & 88.9 & 91.4 \\
Ceftazidime & 73.7 & 62.5 & 69.0 & 44.4 & 68.1 \\
Piperacillin/tazobactam & 70.7 & 66.1 & 65.5 & 51.9 & 67.4 \\
Imipenem & 99.4 & 100.0 & 100.0 & 100.0 & 99.6 \\
Meropenem & 100.0 & 100.0 & 100.0 & 100.0 & 100.0 \\
Gentamicin & 75.4 & 76.8 & 89.7 & 59.3 & 77.4 \\
Amikacin & 81.4 & 85.7 & 93.1 & 70.4 & 82.4 \\
Ciprofloxacin & 86.2 & 85.7 & 89.7 & 51.9 & 83.2 \\
Gatifloxacin & 88.6 & 89.3 & 93.1 & 59.3 & 86.4 \\
\hline
\end{tabular}

${ }^{a}$ BSI: bloodstream infection.

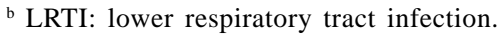

' WSSTI: wound or skin and soft tissue infection

${ }^{\mathrm{d}}$ UTI: urinary tract infection. 
Table 11. Comparison of the antimicrobial susceptibility of S. aureus isolates collected from various infection sites

\begin{tabular}{lcccc}
\hline Antimicrobial agents & \multicolumn{4}{c}{ \% Susceptible Strains } \\
\cline { 2 - 5 } & ${\text { BSI } \mathbf{I}^{\mathbf{a}} \mathbf{( 4 7 3 )}}$ & LRTI $^{\mathbf{b}} \mathbf{( 1 7 3 )}$ & WSSTI $^{\mathbf{c}} \mathbf{( 1 9 7 )}$ & All sites (852) \\
\hline Oxacillin & 70.6 & 52.0 & 66.0 & 66.0 \\
Cefepime & 70.6 & 52.0 & 66.0 & 66.0 \\
Ceftriaxone & 70.6 & 52.0 & 66.0 & 66.0 \\
Ceftazidime & 55.1 & 44.4 & 49.3 & 51.8 \\
Clindamycin & 70.8 & 52.0 & 68.5 & 66.5 \\
Trimethoprim / & 72.7 & 53.6 & 67.5 & 68.0 \\
$\quad$ Sulfametoxazole & & & & \\
Ciprofloxacin & 70.6 & 50.3 & 33.0 & 65.6 \\
Gatifloxacin & 89.0 & 89.0 & 89.8 & 89.3 \\
Teicoplanin & 99.6 & 100.0 & 100.0 & 99.8 \\
Vancomycin & 100.0 & 100.0 & 100.0 & 100.0 \\
\hline
\end{tabular}

${ }^{a}$ BSI: bloodstream infection.

${ }^{b}$ LRTI: lower respiratory tract infection.

${ }^{c}$ WSSTI: wound or skin and soft tissue infection.

Table 12. Comparison of the antimicrobial susceptibility of chromosomally inducible b-lactamase producing isolates collected from various infection sites

\begin{tabular}{lcc}
\hline Antimicrobial agents & & \% Susceptible \\
\cline { 2 - 3 } & $\begin{array}{c}\text { All isolates }^{\mathbf{a}} \\
(\mathbf{4 4 2 . 0 )}\end{array}$ & $\begin{array}{c}\text { Ceftazidime-resistant strains }^{\mathbf{a}} \\
\mathbf{( 1 2 0 . 0 )}\end{array}$ \\
\hline Cefepime & 92.5 & 80.0 \\
Ceftazidime & 72.9 & - \\
Piperacillin/tazobactam & 68.6 & 15.8 \\
Imipenem & 99.3 & 100.0 \\
Meropenem & 100.0 & 100.0 \\
Gentamicin & 71.3 & 42.5 \\
Amikacin & 79.0 & 51.7 \\
Ciprofloxacin & 76.5 & 50.8 \\
Gatifloxacin & 80.3 & 58.3 \\
\hline
\end{tabular}

${ }^{a}$ Include Enterobacter spp., Serratia spp., Citrobacter spp., Providencia spp., Proteus vulgaris, and Morganella morganii. 
$\mathrm{MIC}_{90},>8 \mathrm{mg} / \mathrm{mL}$ for both imipenem and meropenem; Table 2). Tetracycline showed some in vitro activity (64.7\% susceptibility). The next most active compound was tobramycin, which inhibited $56.3 \%$ of the isolates tested $\left(\mathrm{MIC}_{50}, 4 \mathrm{mg} / \mathrm{mL}\right)$. Interestingly, gentamicin $\left(\mathrm{MIC}_{50}, 16 \mathrm{mg} / \mathrm{mL} ; 49.6 \%\right.$ susceptibility) showed higher in vitro activity than amikacin $\left(\mathrm{MIC}_{50},>32 \mathrm{mg} / \mathrm{mL} ; 31.7 \%\right.$ susceptibility). All other compounds were active against $\leq 40 \%$ of the isolates (Table 2).

The results of the antimicrobial susceptibility of three most frequently isolated Gram-positive cocci are shown on Table 5. In general, $34 \%$ of $S$. aureus isolates were resistant to oxacillin. The broad-spectrum b-lactams showed similar spectrum to that shown by oxacillin, except for ceftazidime, which was active against only $51.8 \%$ of $S$. aureus isolates. In addition, crossresistance to other antimicrobial classes was very common among oxacillin-resistant strains (Tables 5 and 6). Clindamycin, trimethoprim/sulfamethoxazole, ciprofloxacin, and gentamicin were active against less than $10 \%$ of oxacillin-resistant S. aureus (ORSA) (Table 6). On the other hand, 69.3\% of ORSA isolates were susceptible to the new quinolone gatifloxacin $\left(\mathrm{MIC}_{90}, 4 \mathrm{mg} / \mathrm{mL}\right)$. Vancomycin was uniformly active against $S$. aureus strains; however, reduced susceptibility to teicoplanin and quinupristin/dalfopristin was detected in 1 isolate (Table 6).

Resistance rates to b-lactams were much higher among CoNS and less than $20 \%$ of the strains were considered susceptible to oxacillin and broadspectrum b-lactams (Table 5). Gatifloxacin was active against $92.7 \%$ of the CoNS isolates $\left(\mathrm{MIC}_{90}\right.$, $2 \mathrm{mg} / \mathrm{mL}$ ) and decreased susceptibility to vancomycin was not detected among CoNS ( $\mathrm{MIC}_{90}$, $2 \mathrm{mg} / \mathrm{mL}$ ). The vast majority of enterococci isolates were represented by E. faecalis and only 1 isolate with reduced susceptibility to glycopeptides was detected during the period of the program (Table 5). In addition, ampicillin resistance was very low among enterococci ( $98.0 \%$ susceptibility). On the other hand, almost $30 \%$ of the enterococci isolates evaluated showed high level resistance to gentamicin or streptomycin (Table 5).
Tables 7 and 8 show the antimicrobial susceptibility of the most frequently isolated species from BSI, while Tables 9, 10, and 11 exhibit the antimicrobial susceptibility of specific species ( $P$. aeruginosa, Enterobacter spp., and S. aureus, respectively) according to the site of infection. Table 12 shows the antimicrobial susceptibility of chromosomally inducible b-lactamase producing strains (Enterobacter spp., Serratia spp., Citrobacter spp., Proteus vulgaris, Morganella morganii, and Providencia spp.) broken down according to the ceftazidime susceptibility.

\section{Discussion}

As resistance to antibiotics continues to increase, surveillance has become a well-recognized necessity, and should combine local, national, and international efforts. Multicenter surveillance program monitoring at the hospital, or even at ward level, is also critical to understanding the relative importance of risk factors, the evolution of resistance over time, as well as for the development and assessment of preventive measures $[1,6]$.

Gram-negative bacilli and $S$. aureus were the predominant pathogens causing infections in the Brazilian medical centers participating in the SENTRY. Since the isolates were consecutively collected according to the site of infection, our results show the most frequently isolated species in the sites of infection evaluated. The rank order of occurrence differed slightly from that reported for North American centers participating in SENTRY [1]. In general, the most important discrepancies were a higher prevalence of Acinetobacter spp. and a lower prevalence of Enterococcus spp. in Brazil, when compared to North American centers. The top 10 pathogens in the present study accounted for almost $90 \%$ of all isolates. Among isolates from BSI, $38.3 \%$ were due to Gram-positive cocci, but Enterococcus spp. was isolated in only $2.7 \%$ of the cases. In contrast, in the U.S., the Gram-positive cocci were responsible for $46 \%$ of the BSI cases and Enterococcus spp. was isolated in almost $10 \%$ of cases [1]. 
The resistance rates were generally higher in Brazil when compared to those of North America, especially among the Gram-negative rods [7, 8]. The main resistance problems detected in this study were: 1 ) extremely high rates of ESBL-producing Enterobacteriaceae; 2) carbapenem resistance among non-fermentative Gram-negative bacilli, especially $P$. aeruginosa; 3 ) and high rates of stably derepressed Bush-Jacoby-Medeiros group 1 strains among Enterobacteriaceae.

The epidemic rate of ESBL-producing strains of $K$. pneumoniae and E. coli in Brazil is of great concern. Although the rates of ESBL-producing strains may vary significantly from region to region, or even from hospital to hospital within the same geographic region, the Brazilian rates are much higher than rates seen in most other parts of the world. In the U.S., ESBL-production rates are usually less than $5 \%$ for K. pneumoniae and less than $2 \%$ for $E$. coli [1]. In Europe the prevalence of ESBL-producing $K$. pneumoniae may be as high as $15 \%$ to $20 \%$, or even higher in isolates from ICUs [9, 10]. In contrast, in the present study, approximately $9 \%$ of E. coli and $50 \%$ of $K$. pneumoniae were characterized as ESBL producers. Other Brazilian studies have validated the SENTRY results by showing similar rates of ESBL-producing strains in several hospitals around the country [11]. Although the extensive dissemination of a plasmid harboring the ESBL gene cannot be completely disregarded, the great genomic variability demonstrated by the ESBL-producing strains strongly suggests the continued selection of resistant mutants [12,13]. Some studies have shown that among other identified risk factors, the use of third-generation cephalosporins, especially ceftazidime, is related to the appearance of ESBL-producing strains [14, 15].

Although these enzymes are usually inhibited by blactamase inhibitors, such as clavulanic acid and tazobactam, a significant percentage of ESBLproducing strains showed in vitro resistance to blactam-b-lactamase inhibitor combinations, including piperacillin/tazobactam. A low activity of b-lactam-blactamase inhibitor combinations against ESBLproducing strains has been documented in other studies and the mechanisms of resistance involved are not completely elucidated [16-18]. Thus, clinical studies are necessary to clarify the role of b-lactam-b-lactamase inhibitor combinations in the treatment of infections due to ESBL-producing strains. Actually, some authors have advocated that the b-lactamase-inhibitor combinations might be used to prevent the emergence of ESBL-producing strains. In a case control-control study conducted in an intensive care unit to investigate the risk acquisition of ESBL-producing strains, blactamase-inhibitor therapy was shown to be a protective factor [15]. Other investigators have been able to decrease the rate of colonization with ESBLproducing K. pneumoniae by using piperacillin/ tazobactam, rather than ceftazidime, in the empirical treatment for nosocomial infections $[15,19]$.

In summary, Table 4 shows that there are very few therapeutic options to treat infections due to ESBLproducing strains in Brazil. Only the carbapenems were active against $>75 \%$ of ESBL-producing $E$. coli, while the quinolones represent a reasonable alternative to the carbapenems against $K$. pneumoniae. All quinolones evaluated inhibited more than $90 \%$ of ESBL-producing K. pneumoniae with similar in vitro activity. In addition, the finding of an isolate of K. pneumoniae with intermediate MIC for meropenem is of great concern since widespread carbapenem resistance among Enterobacteriaceae would have catastrophic consequences.

The antimicrobial susceptibility results for Grampositive cocci showed very high rates of oxacillin resistance among staphylococci with cross-resistance to most antimicrobial agents, except the glycopeptides. On the other hand, our study showed very low rates of resistance to ampicillin and glycopeptides among enterococci. The high rate of susceptibility to ampicillin may reflect the higher prevalence of $E$. faecalis as the cause of enterococci infections in the hospitals evaluated. These results are different from those reported by the SENTRY in the U.S., where both the proportion of E. faecium and the prevalence of glycopeptide resistance are much higher [2].

Isolates from BSI were analyzed separately because they are more likely to represent isolates from true infections and because the number of isolates from this 
site was significantly higher. The antimicrobial susceptibilities of isolates from BSI (Tables 7 and 8 ) were very similar to that found when all isolates were analyzed together (Tables 2 and 5). One of the highest discrepancies was noted in the carbapenem susceptibility of $P$. aeruginosa. While $82.0 \%$ of isolates from BSI were susceptible to imipenem (Table 7), less than $70 \%$ of all $P$. aeruginosa evaluated was susceptible to this compound (Table 2). A tendency of higher rates of resistance among isolates from UTI was also noted for this pathogen. In contrast, the highest rates of susceptibilities were detected among isolates from BSI (Table 9). Similarly, S. aureus from BSI presented higher rates of susceptibility when compared to $S$. aureus from LRTI (Table 11).

Resistance rates to third-generation cephalosporins and b-lactam-b-lactamases inhibitor combinations were high among Enterobacter spp. and other species that produce large amounts of inducible Bush-Jacoby-Medeiros group 1 (AmpC) b-lactamase (Table 10)[20]. More than 440 isolates of Enterobacter spp., Serratia spp., Citrobacter spp., Proteus vulgaris, Morganella morganii, and Providencia spp. were analyzed as a group and the antimicrobial susceptibility pattern of this group was very similar to that showed by Enterobacter spp. (Table 12). When the ceftazidime-resistant strains (stably derepressed Bush-Jacoby-Medeiros group 1) were analyzed separately (120 isolates), we verified that only the carbapenems (100\% susceptibility) and cefepime (80\% susceptibility) showed reasonable activity against this group of multiresistant organisms. The newer quinolones gatifloxacin and levofloxacin were active against $57.5 \%$ of the isolates $\left(\mathrm{MIC}_{50}, 2 \mathrm{mg} / \mathrm{mL}\right.$ ), and all other compounds were active against less than $50 \%$ of the strains (Table 12).

SENTRY is one of the largest and most comprehensive surveillance programs in place worldwide. This program has been contributing to the better understanding of antimicrobial resistance in several areas of the world. Due to the lack of regional data, Brazilian physicians relied on susceptibility data from the U.S. and Europe to guide their empiric antimicrobial therapy and also to direct infection control measures. SENTRY has analyzed more than 4,000 clinical isolates $(3,728$ from nosocomial infections and 612 isolates from community acquired infections) from four Brazilian cities in 3 consecutive years. This Program has detected important differences in the antimicrobial susceptibilities in Brazil, North America, and Europe, further emphasizing the importance of having regional data to guide empiric therapy. In addition, the program has performed molecular typing in several groups of resistant organisms (data not shown). The results from this part of the Program allow us to better understand the mode of dissemination of antimicrobial resistance, and to guide the infection control measures necessary to control the problem [9-11,21].

Antimicrobial resistance may vary significantly within a country and SENTRY evaluate only a small percentage of Brazilian hospitals. However, the leading objective of the Program is to detect the main problems within the country and to guide more regional programs. A broader study that evaluated more than 36 Brazilian hospitals focusing on BSI found that the antimicrobial resistance problems detected by SENTRY are widespread and not restricted to only those hospitals evaluated by the Program [11].

\section{References}

1. Jones R.N., Pfaller M.A. Bacterial resistance: A worldwide problem. Diagn Microbiol Infect Dis 1998;31:379-88.

2. Pfaller M.A., Jones R.N., Doern G.V., et al. Survey of blood stream infections attributable to grampositive cocci: frequency of occurrence and antimicrobial susceptibility of isolates collected in 1997 in the United States, Canada, and Latin America from the SENTRY Antimicrobial Surveillance Program. Diag Microbiol Infect Dis 1999;33:283-97.

3. Sader H.S., Jones R.N., Gales A.C., et al. Antimicrobial susceptibility of patterns for pathogens isolated from patients in Latin American medical centers with a diagnosis of pneumonia: Results from the SENTRY Antimicrobial Surveillance Program (1997). Diag Microbiol Infect Dis 1998;32:289-301. 
4. Sader H.S., Sampaio J.L.M., Zoccoli C., Jones R.N. Results of the SENTRY antimicrobial surveillance program results in three Brazilian medical centers for 1997. Braz J Infect Dis 1999;3:63-79.

5. National Committee for Clinical Laboratory Standards. Methods for dilution antimicrobial susceptibility tests for bacteria that grow aerobically; approved standard fifth edition. Approved document M7-A5. Wayne, Pennsylvania. NCCLS 2000.

6. Guzmán-Blanco M., Casellas J.M., Sader H.S. Bacterial resistance to antimicrobial agents in Latin America. The giant is awakening. Infect Dis Clin North Amer 2000; $14: 67-81$.

7. Diekema D.J., Pfaller M.A., Jones R.N., et al. Trends in antimicrobial susceptibility of bacterial pathogens isolated from patients with bloodstream infections in the USA, Canada and Latin America. SENTRY Participants Group. Int J Antimicrob Agents 2000;13:257-71.

8. Pfaller M.A., Jones R.N., Doern G.V., et al. Bacterial pathogens isolated from patients with bloodstream infection: frequencies of occurrence and antimicrobial susceptibility patterns from SENTRY Antimicrobial Surveillance Program (United States and Canada, 1997). Antimicrob Agents Chemother 1998; 42:1762-70.

9. Babini G.S., Livermore D.M. Antimicrobial resistance amongst Klebsiella spp. collected from intensive care unit in Southern and Western Europe in 1997-1998. J Antimicrob Chemother 2000;42:183-9.

10. Fluit A.C., Jones M.E., Schmitz F.J., et al. Antimicrobial susceptibility and frequency of occurrence of clinical blood isolates in Europe from the SENTRY antimicrobial surveillance program, 1997 and 1998. Clin Infect Dis 2000;30:454-60.

11. Sader H.S. Antimicrobial Resistance in Brazil: Comparison of results from two multicenter studies. Braz J Infect Dis 2000;4:91-9.

12. Gales A. C., Jones R.N., Gordon K.A., et al. Activity and spectrum of 22 antimicrobial agents tested against urinary tract infection pathogens in hospitalized patients in Latin America: report from the second year of the SENTRY Antimicrobial Surveillance Program (1998) J Antimicrob Chemother 2000;45:295-304.
13. Sader H.S., Jones R.N., Winokur P.L., et al. Antimicrobial susceptibility of bacteria causing urinary tract infections in Latin American hospitals: Results from the SENTRY antimicrobial surveillance program (1997). Clin Microbiol Infect 1999;5:478-87.

14. Schiappa D.A., Hayden M.K., Matushek M.G., et al. Ceftazidime-resistant Klebsiella pneumoniae and Escherichia coli bloodstream infection: a case-control and molecular epidemiologic investigation. J Infect Dis 1996; 174:529-36.

15. Piroth L., Aube H., Doise J.M., Vincent-Martin M. Spread of extended-spectrum beta-lactamaseproducing Klebsiella pneumoniae: are betalactamase inhibitors of therapeutic value? Clin Infect Dis 1998; $27: 76-80$.

16. Bryson H.M., Brogden R.N. Piperacillin/tazobactam. A review of its antibacterial activity, pharmacokinetic properties and therapeutic potencial. Drugs 1994;47:506-35.

17. Gales A.C., Bolmströn A., Sampaio J., et al. Antimicrobial susceptibility of Klebsiella pneumoniae producing extended-spectrum b-lactamases (ESBL) isolated in Brazilian hospitals. Braz J Infect Dis 1997;1:196-203.

18. Roland R.K., Mendes R.E., Silbert S., et al. In vitro antimicrobial activity of piperacillin/tazobactam in comparison with other broad-spectrum b-lactams. Braz J Infect Dis 2000;4:226-35.

19. Rice L.B., Eckstein E.C., De Vente J., Shlaes D.D. Ceftazidime-resistant Klebsiella pneumoniae isolates recovered at the Cleveland Department of Veterans Affairs Medical Center. Clin Infect Dis 1996;23:118-24.

20. Bush K., Jacoby G.A., Medeiros A.A. A functional classification scheme for beta-lactamases and its correlation with molecular structure. Antimicrob Agents Chemother 1995; 39:1211-33.

21. Gales A.C., Jones R.N., Sader H.S., SENTRY Study Group. A two year assessment of the pathogen frequency and antimicrobial resistance patterns among organisms isolated from skin and soft tissue infections in Latin American hospitals: results from the SENTRY Antimicrobial Surveillance Program. Int J Infect Dis 2000;4:75-84, 2000. 